\title{
Numerical Calculations for a Boundary Layer Flow past a Moving Vertical Porous Plate with Suction/Injection and Thermal Radiation
}

\author{
Okey Oseloka Onyejekwe, Yohannes Demiss Belete, Nahom Alemseged Worku \\ Computational Mechanics and Dynamics Systems Group, Computational Science Program, Addis Ababa University, Arat Kilo \\ Campus, Addis Ababa, Ethiopia \\ Email: okuzaks@yahoo.com
}

How to cite this paper: Onyejekwe, O.O., Belete, Y.D. and Worku, N.A (2020) Numerical Calculations for a Boundary Layer Flow past a Moving Vertical Porous Plate with Suction/Injection and Thermal Radiation. Applied Mathematics, 11, 172-183. https://doi.org/10.4236/am.2020.113015

Received: January 17, 2020

Accepted: March 8, 2020

Published: March 11, 2020

Copyright $\odot 2020$ by author(s) and Scientific Research Publishing Inc. This work is licensed under the Creative Commons Attribution International License (CC BY 4.0).

http://creativecommons.org/licenses/by/4.0/

\begin{abstract}
The work presented herein investigates the velocity, heat transfer, Nusselt number and skin friction profiles involved in boundary layer flow past a moving vertical porous plate. Similarity transformations are employed to convert the governing nonlinear unsteady momentum and energy equations from their partial differential equation forms to boundary value ordinary differential equations. The resulting equations are then solved numerically by the Runge-Kutta fourth order method with the help of a shooting technique. Several features of the flow and heat transfer characteristics for different values of problem parameters are analyzed and discussed. These include the effects of the radiation parameter $(\mathrm{R})$, suction and injection parameter (c), Grashof (Gr) and Prandtl (Pr) numbers on the flow and heat profiles. Numerical results show the impact of blowing and sucking as well as radation on boundary layer flows of this type. Both the skin frictions as well as the heat transfer rate are also significantly related to the radiation parameter. For all these cases; the numerical results are found to be in agreement with the physics of the problem.
\end{abstract}

\section{Keywords}

Momentum and Energy, Grashof Number, Prandtl Number, Similarity Transformation, Radiation Parameter, Suction, Blowing, Radiation, Porous Plate

\section{Introduction}

Fluid flow and heat transfer for both moving and stationary media have been studied in the past because of its relevance to thermal insulation, waste disposal, 
drying of agricultural products, oil recovery, nuclear reactors, geothermal reservoirs and other practical applications in the areas of engineering and geophysical fields. In addition, cases related to boundary layer flow and heat transfer induced by moving surfaces are vastly significant in extrusion of polymers, glass blowing, metal and fiber spinning. A comprehensive list of relevant work in this area can be found in a monograph by Nield and Bejan [1].

Bejan and Khair [2] studied heat and mass transfer effects for the case of boundary layer flow embedded in porous medium. Related studies involving series expansion, suction and blowing for the case of a vertical plate in a saturated porous medium were carried out by Lai and Kulacki [3] [4] and Raptis [5]. A slightly different study involving not only boundary layer flow but air entrainment over a continuous solid surface moving with a constant velocity in an ambient fluid was carried out by Sakiadis [6]. His work was further extended other investigators such as Ishak et al. [7] [8], Seini and Makinde [9] and Chaturvedi [10].

Magnetohydrodymaics (MHD) of boundary layer flow across a vertical porous plate has a wide area of industrial applications in such areas as geophysics, geothermal energy extractions, astrophysics, plasma studies and crude oil purifications. With reference to these applications, the overriding classical problem in this field was introduced by Blasius [11], who was the first to deal with boundary layer flow over a flat plate. Sakiadis [6] later considered boundary layer flow over a continuously moving solid surface with a constant speed. Erickson et al. [12] later followed this up with different heat and mass transfer considerations including suction and injection.

Soundalgekar and Raman [13] examined alterative aspects of this problem by considering the effects of wall temperature, mass transfer and various other fluid properties. Other considerations by Reddy et al. [14] include MHD boundary layer flow in a channel involving radiation, absorption and heat dissipation and generation.

Other interesting work in this area involves the interaction of MHD boundary layer flow and moving objects. Similarity solutions of MHD flow past a vertical plate with convective boundary condition were carried out by Israel-Cookey et al. [15]. Mostafa and Mahmoud [16] investigated the overall effect of hydromagnetic flow across a moving vertical plate embedded in a porous medium. Ramachandran and Bhaksar [17] extended this study by including other effects such as radiation, and mass transfer. An unsteady MHD boundary layer flow of a rotating fluid past a vertical porous plate was studied by Reddy [18]. He applied a perturbation technique to arrive at the effects of pertinent fluid parameters on fluid flow. Relevant work in this area is reported by Makinde et al. [19] and Pantokratoras [20].

In this paper we consider the effect of suction and injection, thermal radiation and other dimensionless flow variables on boundary layer flow across a moving vertical porous plate. The nonlinear coupled partial differential equation describing the temperature and velocity profiles are transformed into ordinary dif- 
ferential equations using similarity variables and are solved by the fourth order Runge-Kutta shooting technique. The effects of various flow and heat transfer parameters are presented graphically. This includes skin friction coefficient, the Nusselt number as well as the temperature and the velocity profiles. Followed by his is a thorough quantitative description of each of the profiles. This is essential in order to confirm that the solutions obtained are in consonance with the physics which the problem formulations represent.

\section{Problem Formulation}

We consider an unsteady incompressible (constant density) viscous fluid with thermal radiation past a vertical moving porous plate. The $x$-axis is taken as the direction along the plate in the vertical upward direction with the $y$-axis perpendicular to it. Let $u$ and $v$ be the components of the velocity along the $x$ and $y$ axis. The governing equations are represented as

$$
\begin{gathered}
\frac{\partial v}{\partial y}=0 \\
\frac{\partial u}{\partial t}+v \frac{\partial u}{\partial y^{*}}=\mu \frac{\partial^{2} u}{\partial y^{* 2}}+g \beta\left(T-T_{\infty}\right) \\
\frac{\partial T}{\partial t}+v \frac{\partial T}{\partial y^{*}}=\alpha \frac{\partial^{2} u}{\partial y^{* 2}}+\frac{\alpha}{k} \frac{\partial q}{\partial y^{*}}
\end{gathered}
$$

The above equations represent the continuity, momentum and energy equations respectively. The parameter $\mu$ is the kinematic viscosity, $k$ is the thermal conductivity. $\beta, q_{r}, g, T, t, T_{\infty}$; are the volumetric expansion, radiative heat flux, gravity, temperature, time, and wall temperature respectively. We apply Roseland approximation, to represent the radiative heat flux as:

$$
q_{r}=-\frac{4 \sigma}{3 s} \frac{\partial T^{4}}{\partial y^{*}}
$$

where $\sigma, s$ are the Stefan-Boltzmann constant and the mean absorption. Taking the temperature differences to be small, we use the Taylor series expansion, to linearize $T^{4}$ about $T_{\infty}$ to yield:

$$
T^{4}=4 T_{\infty}^{3} T-3 T_{\infty}^{4}
$$

Given Equation (4) and Equation (5) Equation (3) can now be written as:

$$
\frac{\partial T}{\partial t}+v \frac{\partial T}{\partial y^{*}}=\alpha \frac{\partial^{2} T}{\partial y^{* 2}}+\frac{16 \sigma T_{\infty}^{3}}{3 s k} \frac{\partial T}{\partial y^{*}}
$$

The boundary conditions for the velocity and temperature fields at the plate surface and far into the fluid are given as:

$$
\begin{gathered}
u=U_{0}, T=T_{w} \text { at } y^{*}=0 \\
u \rightarrow 0, \quad T \rightarrow T_{\infty} \text { as } y^{*} \rightarrow \infty
\end{gathered}
$$

where $T_{w}, T_{\infty}$ are the wall and ambient temperatures. In order to write the governing equations and the boundary conditions in dimensionless forms the fol- 
lowing dimensionless variables need to be introduced.

$$
s=2 \sqrt{v t}, y=y^{*} / \sqrt{v t}, u=U_{0} f(y), \quad \theta(y)=\left(T-T_{\infty}\right) /\left(T_{w}-T_{\infty}\right)
$$

Substituting Equation (9) into Equation (2) and Equation (3) yields the following nonlinear coupled ordinary differential equations:

$$
\begin{aligned}
& f^{\prime \prime}=-2(c+y) f^{\prime}-G r \theta \\
& \theta^{\prime \prime}=-\frac{2 P r}{(1+R)}(c+y) \theta^{\prime}
\end{aligned}
$$

where

$$
R=\frac{16 \sigma \alpha T_{\infty}^{3}}{3 k K}, G r=\frac{g \beta\left(T-T_{w}\right) s^{2}}{v U_{0}}, \operatorname{Pr}=\frac{\mu}{\alpha}
$$

$y$ is the similarity variable, $f(y)$ is the stream function, $f^{\prime}(y), \theta(y)$ are the dimensionless velocity and temperature.

The corresponding non-dimensional boundary conditions are given as:

$$
f(0)=\theta(0)=1, \quad f(\infty)=\theta(\infty)=0
$$

For the type of boundary layer flow under consideration, both the Nusselt number and the skin friction coefficient are important flow parameters and are described as follows:

$$
\tau_{w}=\left[\mu \frac{\partial u}{\partial y}\right]_{y=0}=\frac{\tau_{w} s}{\mu U_{0}}=f(0)
$$

where $\tau_{w}$ is the shear stress along the plate. The temperature field facilitates the computation of the heat transfer coefficient and is expressed non-dimensionally as the Nusselt number.

$$
N u=\frac{q_{r} s}{k\left(T_{w}-T_{\infty}\right)}=-\theta(0)
$$

The shooting method can be applied to solve the Equation (10) and Equation (11) by replacing each of them by two first order ODEs with known functions (Dirichlet or Neumann). Nonlinear terms do not pose any special problem with the shooting technique; however a good initial guess is helpful. The following equations further elucidate the application of this scheme for this study. For Equation (10)

$$
\begin{gathered}
P=f^{\prime}, \quad f(0)=1, \quad w=P^{\prime}=f^{\prime \prime}=-2(c+y) f^{\prime}, \\
z=\theta^{\prime}, \quad \theta(0)=1, \quad m=z^{\prime}=\theta^{\prime \prime}=-\frac{2 P r}{(1+R)}(c+y) \theta,
\end{gathered}
$$

To solve these system of IVPs (initial value problems), we need $P(0)$ and $z(0)$. i.e. $f^{\prime}(0)$ and $\theta^{\prime}(0)$ which are not given in the boundary conditions. These values should therefore be guessed in order to proceed with the numerical calculations. By applying the shooting Runge Kutta fourth order technique, computed values at $f(1)$ and $\theta(1)$ are checked with those specified ab initio until the differences between the computed and specified differ by a given error to- 
lerance. Adjustments are made by using a secant approximation.

\section{Results and Discussion}

The primary physical quantities of interest here are, the velocity and temperature profiles, skin friction coefficient and the Nusselt number. In order to arrive at a physical insight into the problem formulation a parametric study is carried out to illustrate the effects of the different governing parameters namely the Grashof, Nusselt and Prandtl numbers and the radiation and suction/blowing parameters on fluid flow and heat transfer processes. Throughout the calculation the Prandtl number is fixed at a value of 0.71 (value for air) and the Grashof number is set at 10. All the graphs correspond to these values unless otherwise stated on the appropriate graph. Graphical results for the Nusselt number as well as those for the skin friction in comparison with the radiation parameters are displayed.

Figure 1 shows the dimensionless temperature versus the dimensionless distance. With a constant values of suction parameter, Grashof and Prandtl numbers, it demonstrates the temperature distribution for different values of radiation parameter $R$. This parameter $(R)$ essentially defines the relative contribution of conduction heat transfer to thermal radiation heat transfer. Given this, it not surprising that the temperature values increase as the radiation parameter increases. In this case, the fluid is heated, there is a reduction of the heat transfer from the wall followed by a rise in temperature.

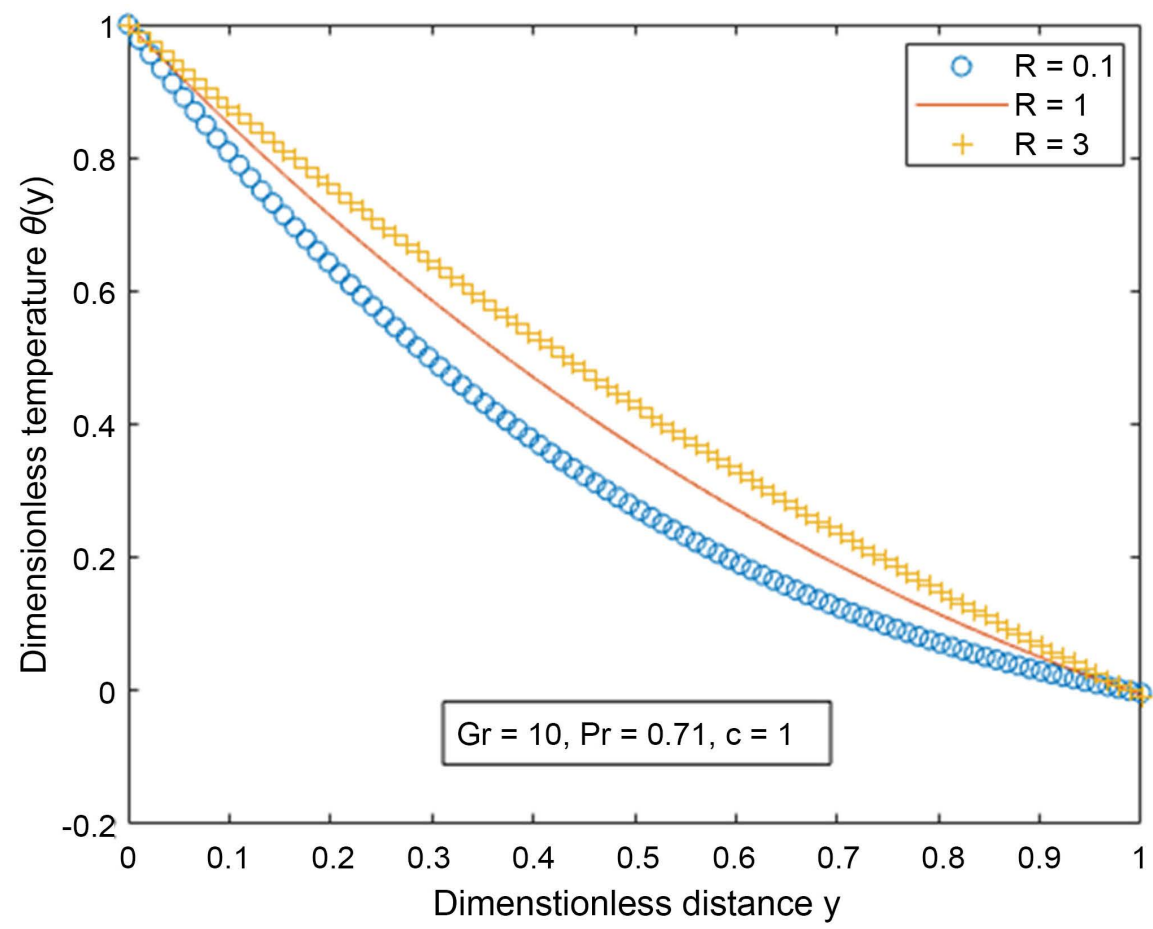

Figure 1. Temperature $\theta(y)$ versus distance $y$ for different values of radiation parameter $R$ and fixed $c$, Prand $G$. 
The effect of suction on the temperature profile is shown in Figure 2. It can be seen that the temperature decreases with increase in value of the suction parameters while other parameters kept constant. Figure 3 and Figure 4 show that

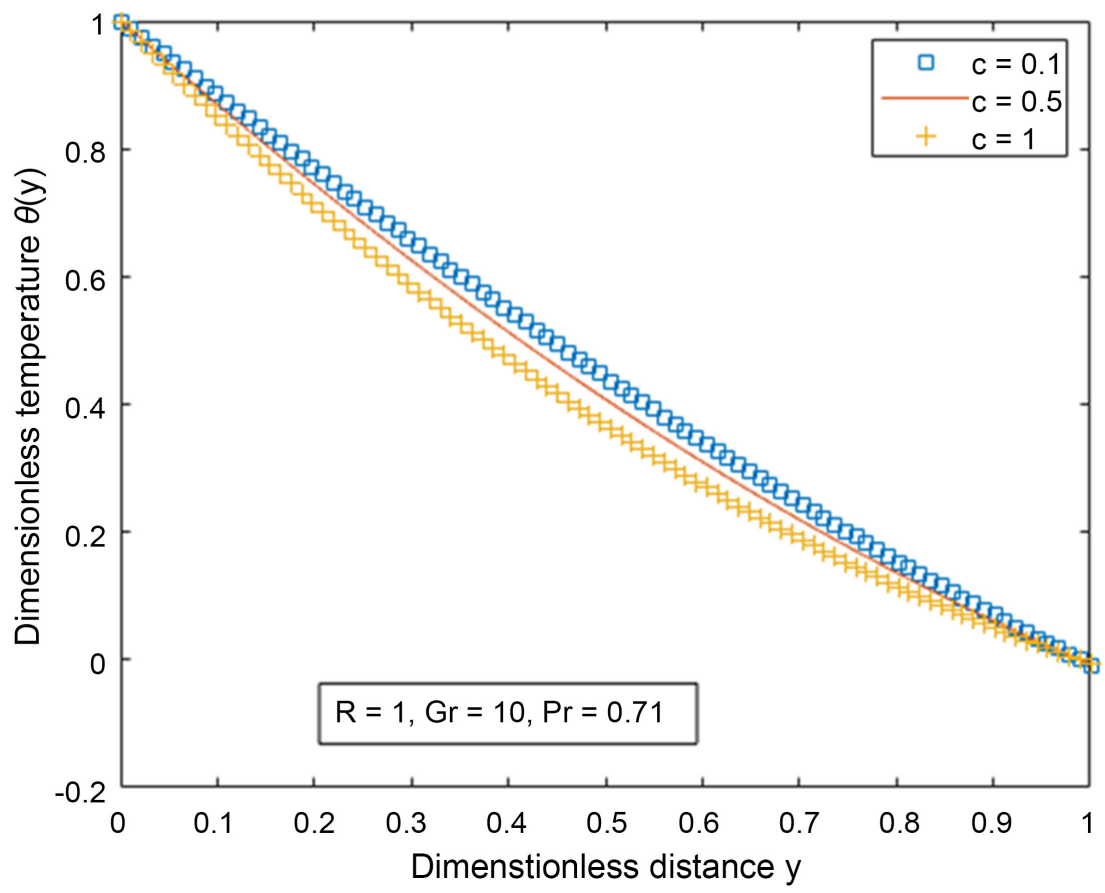

Figure 2. Temperature $\theta(y)$ versus distance $y$ for different values of radiation parameter $c$ and fixed $R, P r$ and $G r$.

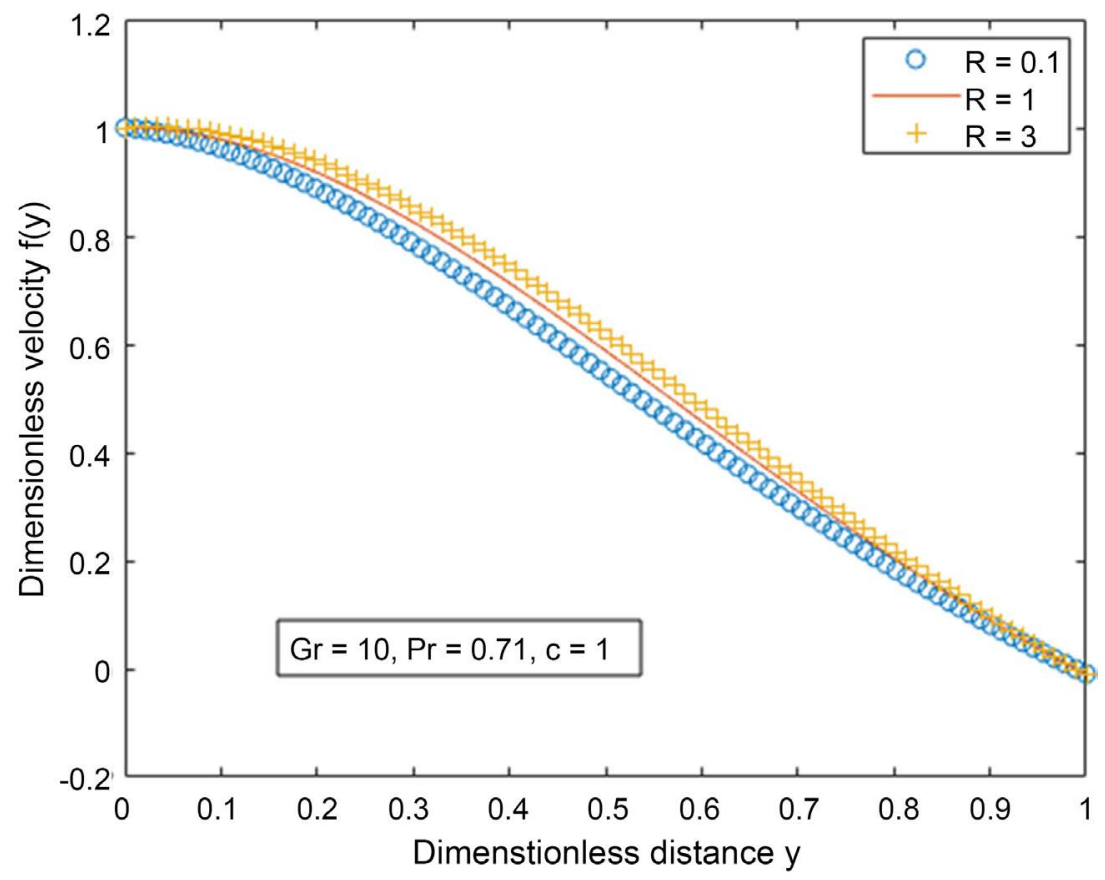

Figure 3. Velocity $f(y)$ versus distance $y$ for different values of radiation parameter $R$ and fixed $c$, Prand $G r$. 


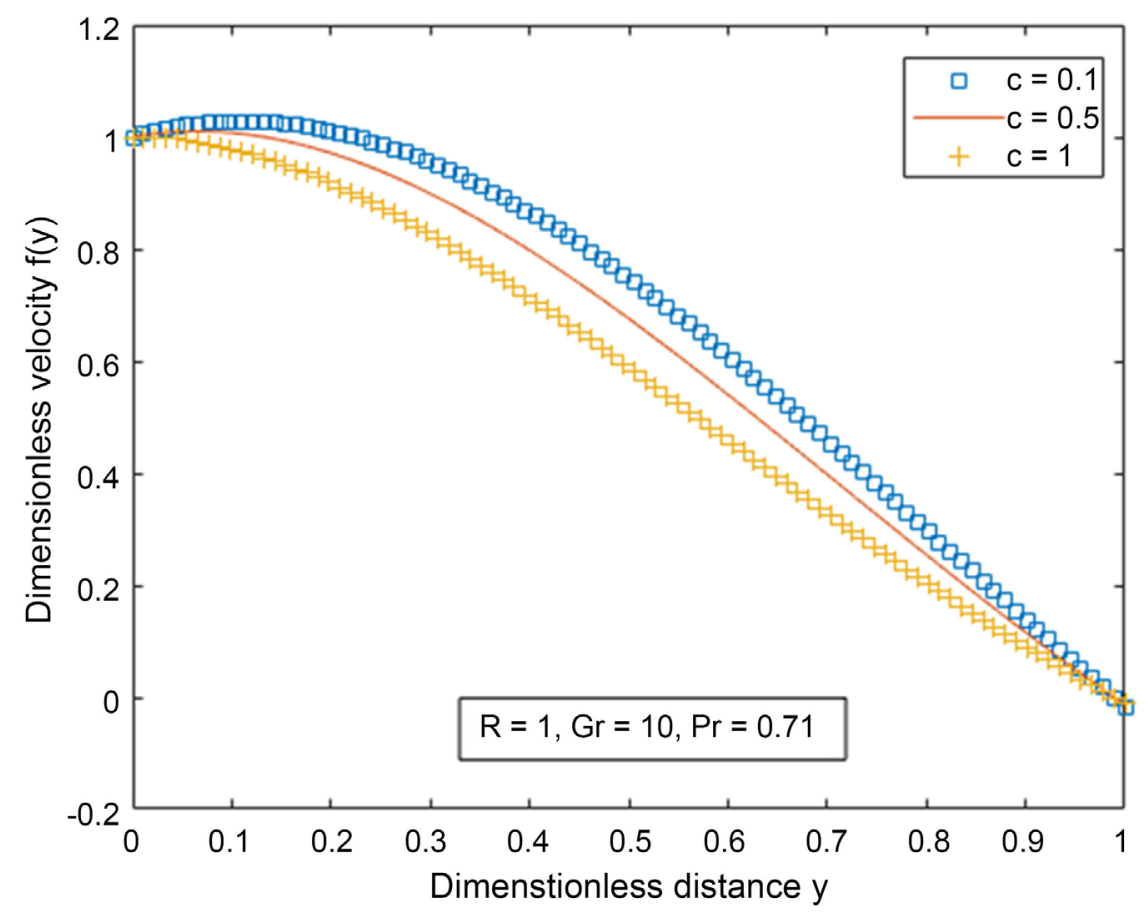

Figure 4. Velocity $f(y)$ versus distance $y$ for different values of radiation parameter $c$ and fixed $R, \operatorname{Pr}$ and $G r$.

suction and radiation parameters have a profound effect on the velocity profiles especially very close to the plate. For example there is a rise in the velocity profile with increase in radiation parameter. The opposite is the case for the suction parameter. Most of these observation happen very close to the plate surface and contribute to the overall shapes of the profiles. It can be observed that maximum velocity occurs a short distance from the plate where the overall effect of the plate movement is the greatest and decreases exponentially farthest from the plate.

On the whole it can be seen that the velocity increases as the mass suction increases. The resultant effect on the momentum boundary layer is to make it thinner On the other hand an increase in mass suction decreases the temperature profile.

Other parameters of interest are the skin friction coefficient and Nusselt numbers. Figure 5 shows that an increase in the radiation parameter $(R)$ and suction brings about a decrease in the wall heat transfer rate with other parameter fixed. The asymptotic shape of the graphs suggests that there is a limiting value of $(R)$ that will not result in any change in the wall heat transfer rate. Figure 6 displays an opposite effect for the skin friction for the same set of conditions. An overall increase in rates of skin friction is displayed for the same parameter combinations.

Figures 7-10 show the effect of fluid or mass injection and suction. into the boundary layer flow for all the flow and heat transfer parameters considered above. Figure 7 and Figure 8 display the contrasting effects of suction and parameter 


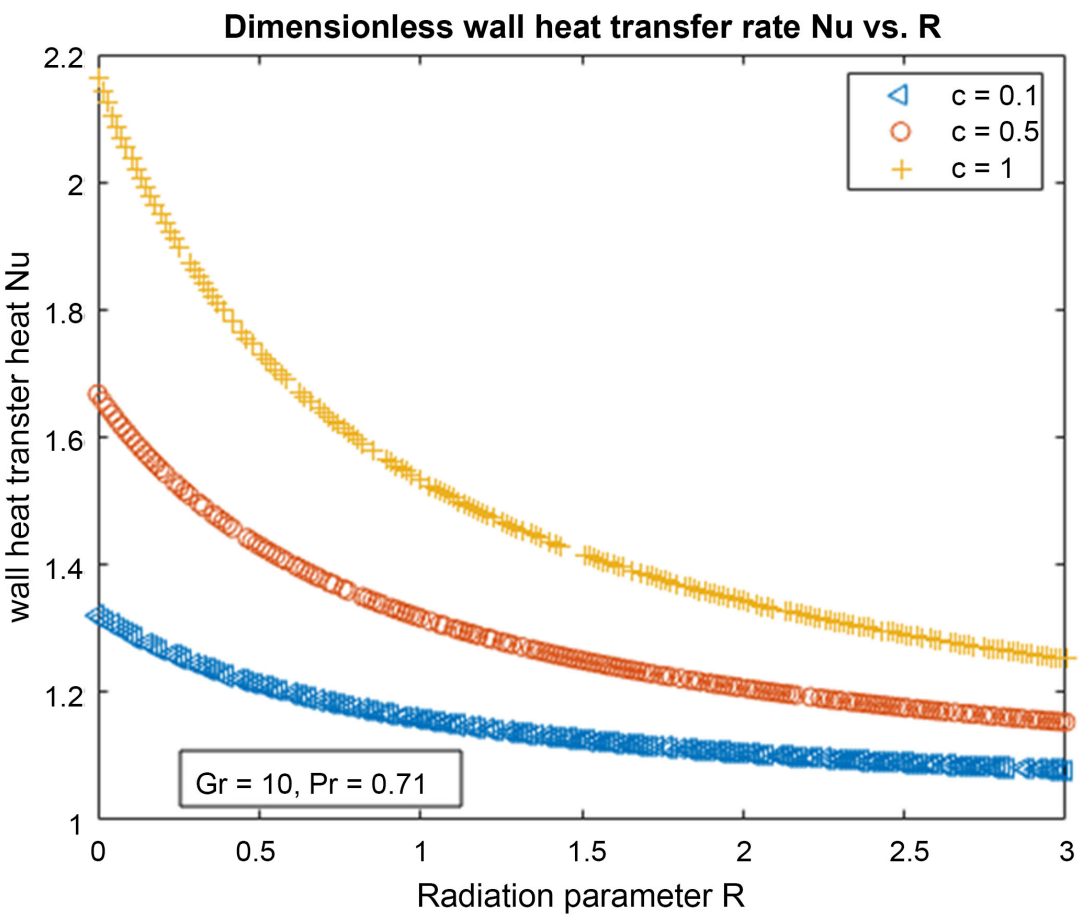

Figure 5. Wall heat transfer rate $-\theta(0)$ versus the Radiation parameter $R$ for fixed $G r$, and $\operatorname{Pr}$ and various values for $c$.

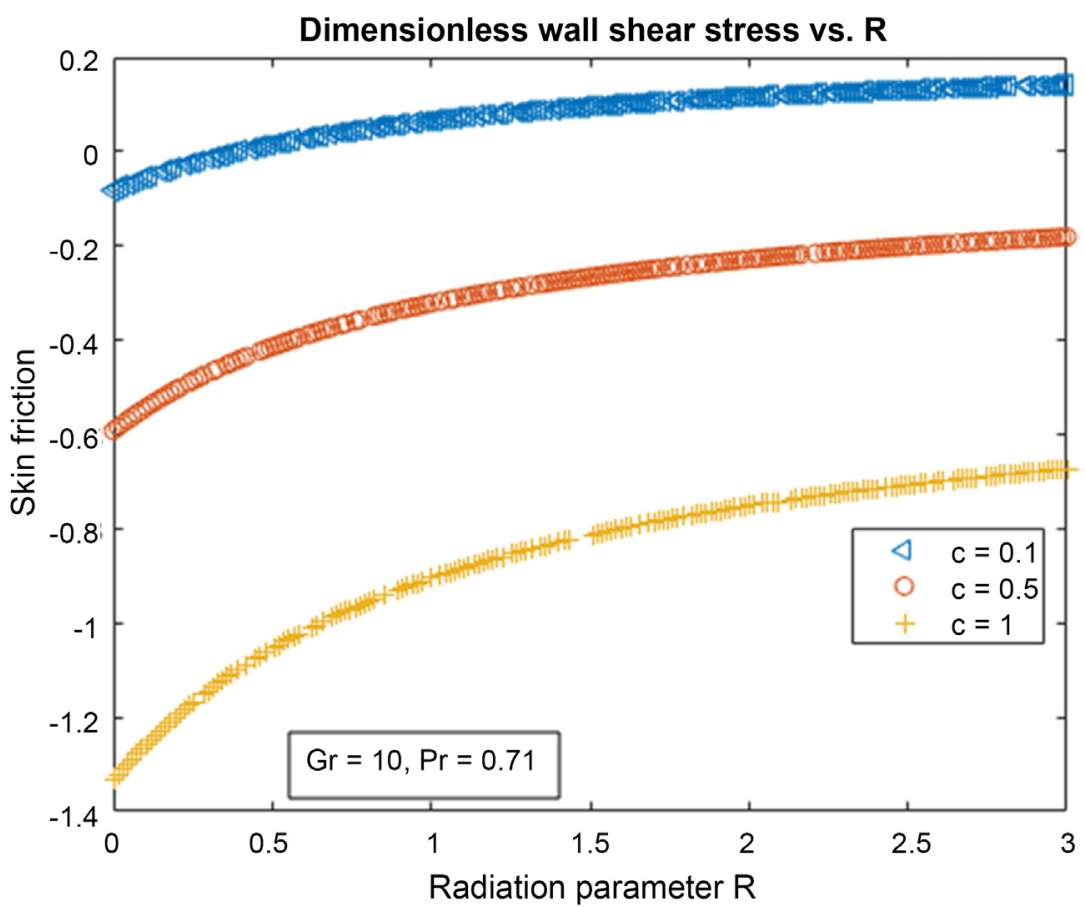

Figure 6. Skin friction $f^{\prime}(0)$ versus the Radiation parameter $R$ for fixed $G r$ and $\operatorname{Pr}$ and various values for $c$ and $R$.

values on the temperature and velocity profiles. While suction reduces the skin friction at the plate injection accomplishes the opposite. Anan increase suction 


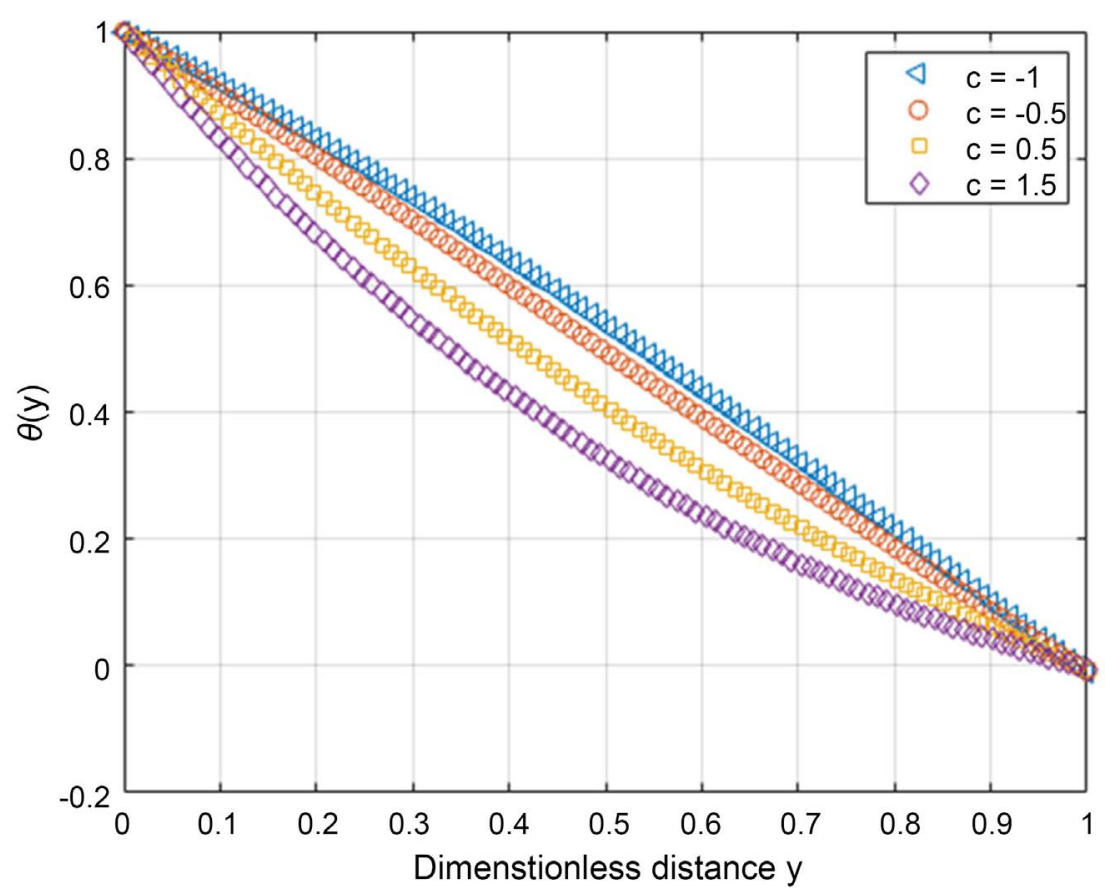

Figure 7. Temperature $\theta(y)$ versus distance $y$ for different values of radiation parameter $c$ and fixed $R, P r$ and $G r$.

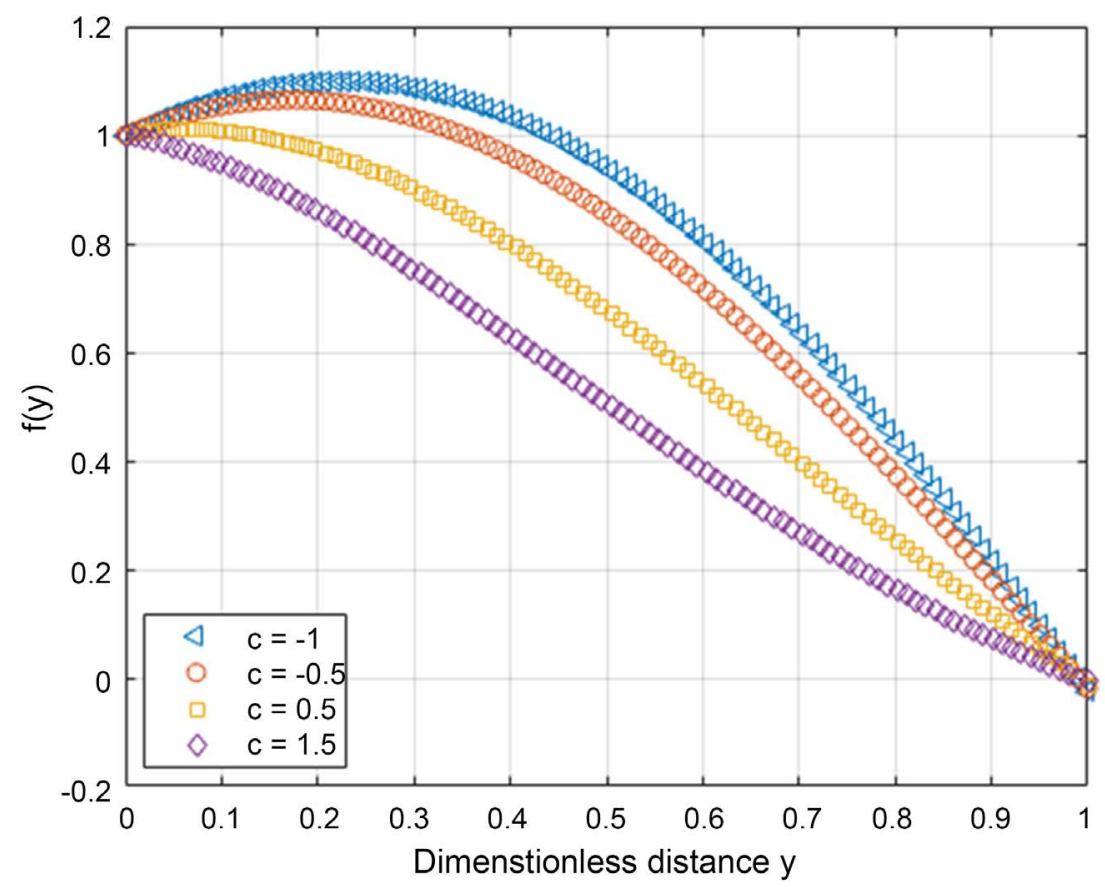

Figure 8. Velocity $f(y)$ versus distance y for different values of $c$ involving suction and blowing and fixed $R, \operatorname{Pr}$ and $G r$.

and radiative heat transfer result in a decrease in the heat transfer on the plate. Figure 10 shows an opposite effect for the case of fluid injection. The same trend is observed in Figure 9. 


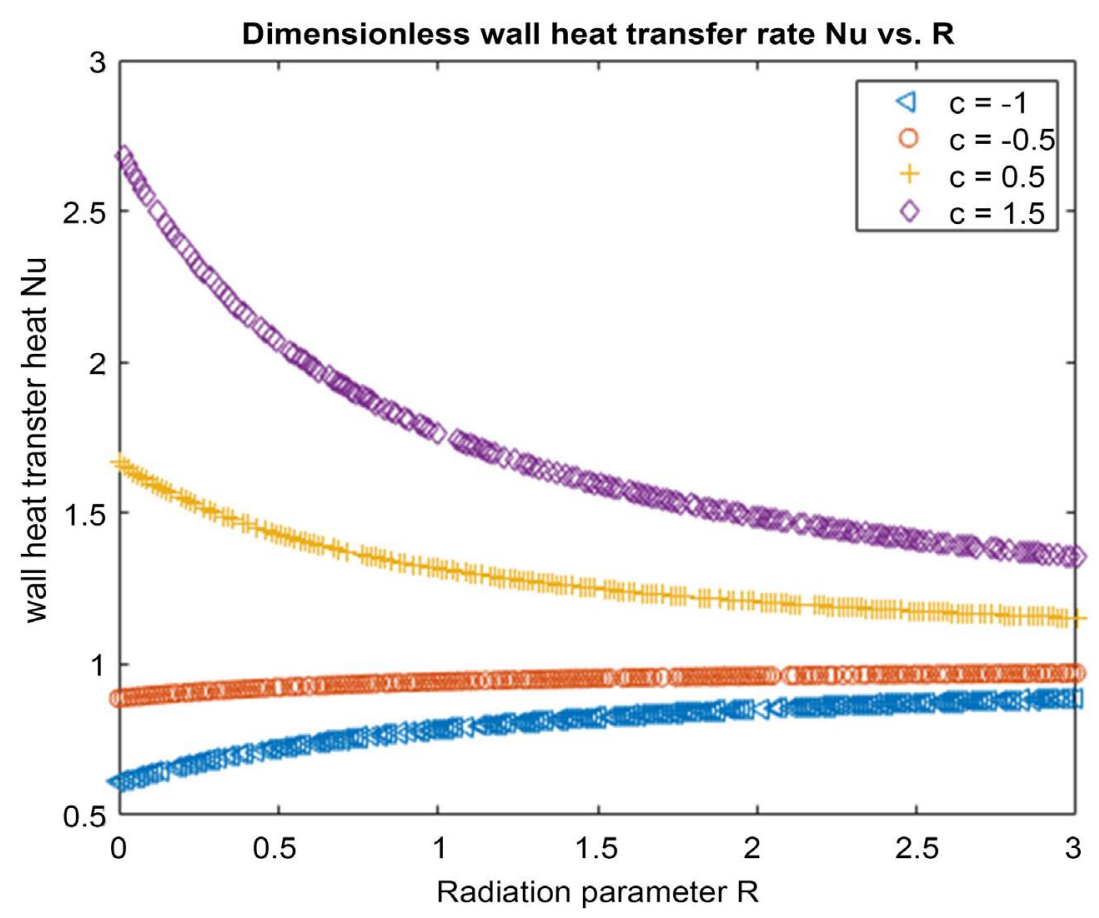

Figure 9. Wall heat transfer rate $-\theta^{\prime}(0)$ vs $R$ for different values of $c$ involving suction and injection with fixed $G r$ and $P r$.

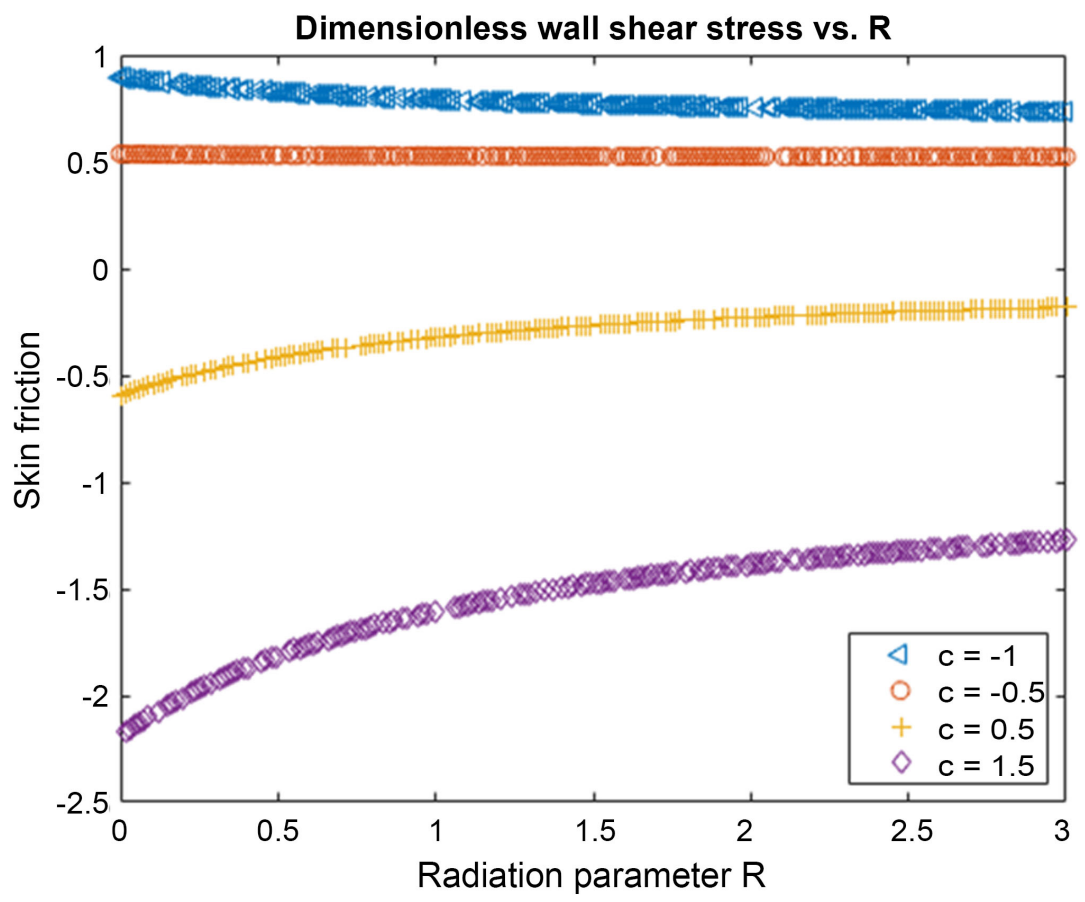

Figure 10. Skin friction $f^{\prime}(0)$ vs $R c$ involving suction and injection with fixed Gr and Pr.

\section{Coclussion}

Numerical computations have been carried out to ascertain the impact of vari- 
ous physical parameters on fluid flow involving a heat transfer process and the effects have been found to be quite significant. There is enough evidence to confirm that boundary layer profiles exist for as many physical considerations as possible. More investigations are further needed to verify the effects of those physical parameters that were not given full considerations in this study given the relevance of this type of flow in industrial applications. For example, the fact that both the radiation and suction parameters have a profound influence on the rate of heat transfer as well as the skin friction coefficient is an observation that evokes further research.

\section{Acknowledgements}

We express our thanks to the contribution of the anonymous reviewers whose comments significantly improved the quality of this paper.

\section{Conflicts of Interest}

The authors declare no conflicts of interest regarding the publication of this paper.

\section{References}

[1] Nield, D. and Bejan, A. (2013) Convection in Porous Media. Springer, New York. https://doi.org/10.1007/978-1-4614-5541-7

[2] Bejan, A. and Khair, K.R. (1985) Heat and Mass Transfer by Natural Convection in a Porous Medium. International Journal of Heat and Mass Transfer, 28, 909-918. https://doi.org/10.1016/0017-9310(85)90272-8

[3] Lai, F.C. and Kulacki, F.A. (1990) Coupled Heat and Mass Transfer from a Sphere Buried in an Infinite Porous Medium. International Journal of Heat and Mass Transfer, 33, 209-215. https://doi.org/10.1016/0017-9310(90)90155-N

[4] Lai, F.C. and Kulacki, F.A. (1991) Coupled Heat and Mass Transfer from by Natural Convection from Vertical Surfaces in a Porous Medium. International Journal of Heat and Mass Transfer, 34, 1189-1194. https://doi.org/10.1016/0017-9310(91)90027-C

[5] Raptis, A. (1998) Radiation and Free Convection Flow through a Porous Medium. International Communications in Heat and Mass Transfer, 25, 289-295. https://doi.org/10.1016/S0735-1933(98)00016-5

[6] Sakiadis, B.C. (1961) Boundary Layer Behavior on a Continuous Solid Surface. AIChE Journal, 7, 221-225. https://doi.org/10.1002/aic.690070211

[7] Ishak, A., Nazar, R. and Pop, I. (2007) Boundary Layer on a Moving Wall with Suction and Injection. Chinese Physics Letters, 24, 2274-2276. https://doi.org/10.1088/0256-307X/24/8/033

[8] Ishak, A., Nazar, R. and Pop, I. (2009) Flow and Heat Transfer Characteristics on a Moving Flat Plate in a Parallel Stream with Constant Heat Flux, Heat Mass Transfer. Chinese Physics Letters, 45, 563-567. https://doi.org/10.1007/s00231-008-0462-9

[9] Seini, Y.I. and Makinde, O.D. (2013) MHD Boundary Layer Flow Due to Exponential Stretching Surface with Radiation and Chemical Reaction. Mathematical Problems in Engineering, 2013, Article ID: 163614. https://doi.org/10.1155/2013/163614

[10] Chaturvedi, N. (1996) On MHD Flow past an Inclined Porous Plate with Variable 
Suction. Energy Conversion and Management, 37, 623-627. https://doi.org/10.1016/0196-8904(95)00199-9

[11] Blasisus, H. (1908) Zeitschriftfur Mathematik Physik. Volume 56.

[12] Erickson, L.E., Fan, L.T. and Fox, V.G. (1966) Heat and Mass Transfer on a Moving Continuous Flat Plate with Suction and Injection. Industrial \& Engineering Chemistry Fundamentals, 5, 19-25. https://doi.org/10.1021/i160017a004

[13] Soundalgekar, V.M. and Raman, T.V. (1980) Heat Transfer in Flow past a Continuous Moving Plate with Variable Temperature. Heat and Mass Transfer, 14, 1-93. https://doi.org/10.1007/BF01806474

[14] Reddy, S.H., Raju, M.C. and Reddy, E.K. (2016) Radiation Absorption and Chemical Reaction Effects on MHD Flow of a Heat Generating Casson Fluid Past Oscillating Vertical Porous Plate. Frontiers in Heat and Mass Transfer, 7, 21. https://doi.org/10.5098/hmt.7.21

[15] Israel-Cookey, C., Ogulu, A. and Omubo Pepple, V.B. (2003) Influence of Viscous Dissipation on Unsteady MHD Free-Convection Flow Past an Infinite Vertical Plate in Porous Medium with Time-Dependent Suction. International Journal of Heat and Mass Transfer, 46, 2305-2311. https://doi.org/10.1016/S0017-9310(02)00544-6

[16] Mostafa, A. and Mahmoud, A. (2007) Variable Viscosity Effects on Hydromagnetic Boundary Layer Flow along a Continuously Moving Vertical Plate. Applied Mathematical Sciences, 1, 799-814.

[17] Ramachandra, P.V. and Bhaksar, R.N. (2007) Radiation and Mass Transfer Effects on Unsteady MHD Free Convection Flow Past a Heated Vertical Plate in a Porous medium and with Viscous Dissipation. Theoretical Applied Mechanics, 34, 135-160. https://doi.org/10.2298/TAM0702135P

[18] Reddy, P.G. (2017) MHD Boundary Layer Flow of a Rotating Fluid Past a Vertical Porous Plate. International Journal of Computer and Applied Mathematics, 12, 579-593.

[19] Makinde, O.D. (2005) Free Convection Flow with Thermal Radiation and Mass Transfer past a Moving Vertical Porous Plate. International Journal of Heat and Mass Transfer, 32, 1411-1419. https://doi.org/10.1016/j.icheatmasstransfer.2005.07.005

[20] Pantokratoras, A. (2008) Some Exact Solutions of Boundary Layer Flows along a Vertical Plate with Bouyancy Forces Combined with Lorentz Forces under Uniform Suction. Mathematical Problems in Engineering, 2008, Article ID: 149272. https://doi.org/10.1155/2008/149272 